\title{
Popular health beliefs: Old wives tales about pregnancy and its outcome in Mosul city.
}

\author{
Dhafer B. AL-Youzbaki Nuha Hachim Asma A. AL-Jawadi
}

Department community medicine, College of Medicine, University of Mosul

(Ann. Coll. Med. Mosul 2007; 33(1\&2):42-50)

Received: $18^{\text {th }}$ Feb 2007; Accepted: $10^{\text {th }}$ Sept 2007

\begin{abstract}
:
Background: Searching of popular health beliefs carries many benefits to the whole health process and any physician wants to function effectively in his community must be aware, socially, about these important popular beliefs about health and illness that may manipulate any treatment or preventive health plan. This work was conducted to evaluate the most important popular health beliefs related to pregnancy and its outcome.
\end{abstract}

Methods: A cross sectional design was used in this work. From the sociological point of view, it is a qualitative research, using a consecutive sample of women attendants at a primary health care centers for the period from. $1^{\text {st }}$. October 2006 to $1^{\text {st }}$. February 2007.

Assessment of the popular health beliefs about pregnancy regarding nutrition, hygiene, physical activity, dressing, utilization of medical services during pregnancy, deliveries, breast feeding, and sexual activities throughout the pregnancy was carried out by the use of especially designed questionnaire. All the information were gathered from women more than 40 years old, who have children and living in Mosul city since 30 years.

Results: Most of the participants in this study agreed about the importance of organ and red meats, milk, vegetables and fruits during pregnancy (99\%, 97\%, 95\%, and $94 \%$ respectively). A lower proportion agreed about the usefulness of vitamins $(63 \%)$, iron tablets $(61 \%)$ and white meats $(61 \%)$. Sufficient numbers $(71 \%)$ regarded bathing as a healthy practice during pregnancy as well as tooth brushing (68\%) and hand washing (92\%). Only $36 \%$ went with walking for the pregnant women, $96 \%$ were against aerobics and also against running. More than two thirds $(77 \%)$ supported vaccination, all refused exposure to X-ray and $90 \%$ also refused non-prescribed medication. The majority (91\%) agreed about the importance of hospital deliveries and three quarters $(75 \%)$ disagreed about untrained dia as a birth attendant. Fortunately, $83 \%$ were agreed about giving the colostrums to the new born directly after delivery. A dangerous proportion (18\%) of mothers was assured about the benefits of application ash of dung or dung on the umbilical stump. Breast feeding got support from $84 \%$ while, $60 \%$ agreed about 3 weeks and more as a rest period after delivery.

Conclusion: Many popular health beliefs regarding pregnancy in Mosul city go in agreement with the scientific health facts and theories that should be reinforced and supported. Moreover, the very hazardous popular health belief that ash of dung or dung may be applied to the umbilical stumps of the newly born babies, still needs an intensive intervention health educational program.

\footnotetext{
الخلاصة: إن العديد من المعتقدات الطبية الشعبية فيما يخص الحمل في محافظة نينوى تذهب بالمو افقة مع الحقاك الحقائق

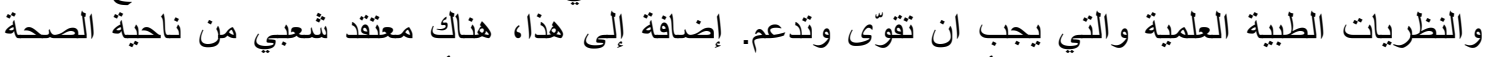

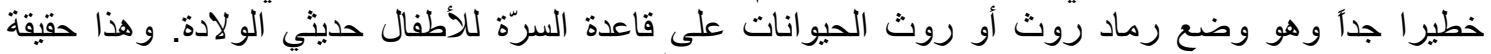

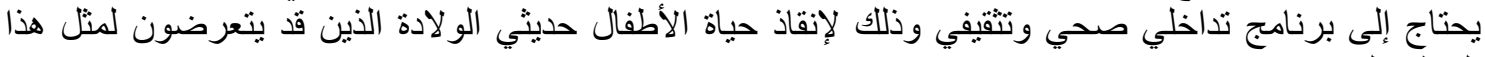


C ulture consists of a body of learned beliefs, traditions, and guides for behaving and interpreting behavior that are shared among members of a particular group. It includes values, beliefs, customs, communication styles, behaviors, practices, and institutions. The visible aspects of a culture include clothing, art, buildings, and food. The less visible aspects include values, norms, worldviews, and expectations. Culture influences an individual's health beliefs, behaviors, activities and medical treatment outcome. Because of the significant influence of culture upon health and related outcome, health care professionals should be culturally aware in order to provide optimum health care to patients ${ }^{(1,2)}$.

Health care sectors include three main domains namely: the professional, the folk(traditional) and the popular health care sector $^{3}$. The professional health sector is the scientific and professional one which is also known as the sector of the modern medicine. The folk(traditional) health care sector is the professional, but non-scientific one, which is usually overlapped with the professional and popular sectors and it may be secular or sacred, or a combination of both. The popular health care sector is the non-scientific and non-professional health care sector. It is the largest part of any health care system and consists of the lay, non-professional, non-specialist arena in which illness is initially defined for an individual and treatment is initiated. ${ }^{(4,5)}$

All cultural and ethnic groups hold concepts related to health and illness and associated practices for maintaining wellbeing or providing treatment when it is indicated. For an individual, socioeconomic, educational, geographic, religious and other factors will mediate and shape cultural and ethnic health beliefs. For this reason, a health care practitioner should be aware of these important beliefs in the community where he is working in order to function effectively. In addition, he should never assume that an individual from one ethnic group holds the same beliefs as another from the same group. Caution is needed to avoid stereotyping. However, there are common elements of belief that may be shared among members of cultural and ethnic groups and a general familiarity with these can be very helpful in further understanding a patient's particular perspective ${ }^{(6,7)}$.
From health education points of views, it is important and crucial to understand the popular health beliefs system in every society in order to evaluate these beliefs whether beneficial, harmful or useless since such information is very important in conducting any health education program. The pregnancy and child rearing are among the most vital biological processes in the human life and understanding these processes culturally and sociologically from all perspectives and through their context is a very important issue to those aiming to practice modern medicine efficiently ${ }^{(8)}$. The aim of the present study is to pin point the most common popular health beliefs about pregnancy and its outcome among a sample of women who attended primary health care centers in Mosul.

\section{Subjects and methods:}

In order to achieve aims of the present study, a mini-cross sectional study design was adopted for this work. More specifically a qualitative research plan (from sociological perspective) was used in order to obtain a consecutive sample of attendants at primary health care centers. The main information were obtained from old wives tales. The inclusion criteria for the participants were:

1. Women more than 40 years.

2. Must have offspring.

3. More than 30 years living inside Mosul city.

Two primary health care centers were chosen from Mosul city, one from the right side (Al- hadbaa training primary health center) and the other from the left side (Alsharqi primary health center). A total sample of 200 respondents $(100 \%$ from each center) was reached (however only 5 women refused to participate in this work and they were excluded), The participants were approached in the waiting room of the primary health care clinics and data collection was done by two trained interviewers using an especially designed questionnaire form in the following manners:

1. The aim of the study was explained to each respondent.

2. The response was obtained in one of three choices as accepted (or beneficial), equivocal, or not accepted (or harmful). Other few questions were aimed to obtain the frequency of usage for certain subject per day or per week. 
The questions that were included in the form were about the following topics:

1. Nutrition: Includes questions about the benefits of the following food: red, white and organ meats; animal and plant oils; carbohydrates, sugars, chocolates and soft drinks; vegetables, milk and fruits; iron and vitamins supplements; nuts; and finally herbs.

2. Hygiene: Includes questions about bathing and tooth brushing with their frequencies, in addition to hand washing.

3. Physical activity: There were questions about walking, running, heavy weight lifting, up stairs climbing and aerobics during pregnancy.

4. Dressing: Tight and wide clothes during pregnancy, high shoes, flat shoes and the color taboo or preference, were asked about.

5. Medical services: Includes questions about vaccination and health visits during pregnancy, the use of prescribed and non-prescribed medication in addition to X-rays exposure during pregnancy. Moreover, their ideas regarding the dentist visits during pregnancy.

6. Contraception: Includes questions about beliefs regarding different modalities of contraception as oral, injected, device or segregation.

7. Delivery: The respondents were asked about the preferable methods and the sites of delivery whether to be undertaken at home by trained or untrained dai or inside hospitals.

8. Umbilical stump care: The interviewers asked about the use of dung, powders contents of medical capsules, medical tablets or ointments as a dressing over umbilical stumps of newly born babies.

9. Infant feeding practice: Such as the preferable feeding practice applied to the newly born babies whether it is breast milk alone, bottle feeding, or mixed types.

10. Post-delivery rest: Includes questions about the favorable periods for rest after delivery.

11. Sexual activity: During the first, second and third trimesters were also questioned.
After completing the questionnaires, the data were analyzed and sorted out by tables using special statistical analysis.

\section{Results:}

The mean age of the study women was 51.78 years. The following are the main results:

Table (1) shows the popular health beliefs about nutrition during the period of pregnancy, where many of these popular health beliefs are quite scientifically true such as (95\%) of the respondents agreed that vegetables are beneficial to women during pregnancy, as well as fruits (94\%), milk $(97 \%)$, vitamins $(63 \%)$, Iron tablets $(61$ $\%)$, organ and red meat (99\%) and white meat $(61 \%)$. On the other hand, this Table indicates unhealthy popular beliefs about diets during pregnancy such as $(93 \%)$ of the respondents agreed that both carbohydrates and sugars are beneficial to the pregnant, as well as animal oil (64\%) and soft drinks $(47 \%)$.

Table (2) indicates that almost two thirds of the respondents $(71 \%)$ regarded bathing as an essential activity to pregnant, but they differ in the preferable frequencies for that activity. The same thing is applied about tooth brushing and hand washing.

Tables (3) shows that despite the popular beliefs about physical activity during pregnancy, the greater part of the respondents disagreed with certain activities such as up-stairs climbing (84 \%), aerobics $(96 \%)$ and heavy weight lifting $(100 \%)$ as well as running $(96 \%)$. While, vague results were gained regarding walking activities during pregnancy.

Table (4) illustrates that the bulk of the respondents $(97 \%)$ agreed with using wide clothes for the pregnant and the wearing of flat shoes (98\%). Again, about two thirds of the respondents $(77 \%)$ refused tight clothes as well as high heal shoes $(68 \%)$. Ambiguous results were got regarding color taboos and color preference for women during pregnancy

Table (5) discloses that just more than three quarters of the respondents $(77 \%)$ agreed with the benefits of vaccination for all pregnants; similar results $(80 \%)$ were obtained regarding prescribed medication. 
On the other hand, all respondents disagreed with the exposure of pregnant women to X-rays in any form in the whole period of pregnancy. Again $90 \%$ refused any non-prescribed medication to the pregnant. Moreover, (62 \%) of the respondents refused even prescribed injections for the pregnant. Indistinct results were achieved regarding health visits plus dentist procedures during the period of pregnancy.

Table (6) confirms that the greater part of the respondents refused the use of any form of contraception except segregation method, where $85 \%$ disagreed with oral contraception, $88 \%$ rejected injectable types on contraception and $62 \%$ said no to the utilization of intra-uterine contraceptive devices.

Table (7) tells the story of the old wives beliefs about the preferable place of delivery and birth attendants. Nearly all the respondents $(91 \%)$ were in agreement with hospital delivery and most of them (83\%) said yes about the use of colostrum in early postnatal days. Seventy five per cent of the respondents disagreed with deliveries that are managed by untrained dai, while unclear results were obtained regarding deliveries that happened at home or by even trained dai.

Table (8) signifies that there is still an important and dangerous proportion (18\%) of the respondents who agreed with application of ash of dung or dung to the umbilical stump of the newly born babies. Significant part of the respondents (59\%) coincided with application of any kind of ointment to the umbilical stumps.

Table (9) demonstrates that most of the respondents $(84 \%)$ accepted the practice of breast feeding, while still there are vague opinions about bottle feeding practice as well as mixed type of feeding to the newly born babies.

Table (10) reveals that almost two thirds (64\%) of the respondents agreed with 2 weeks period of rest. Similar proportion $(60 \%)$ preferred 3 weeks or more period of rest for the pregnant after delivery to return to her normal activities.

Lastly, Table (11) proves that $55 \%$ of the respondents went in agreement with sexual activities for the pregnant in her last trimester, while $48 \%$ did not accept this activity during the first trimester.

Table (1): Popular health beliefs about nutrition during pregnancy

\begin{tabular}{|c|c|c|c|c|c|c|}
\hline \multirow{2}{*}{ Items } & \multicolumn{2}{|c|}{ Agree } & \multicolumn{2}{c|}{ Equivocal } & \multicolumn{2}{c|}{ Disagree } \\
\cline { 2 - 7 } & No. & $\%$ & No. & $\%$ & No. & $\%$ \\
\hline Animal oil & 128 & 64 & 46 & 23 & 26 & 13 \\
\hline Plant oil & 80 & 40 & 64 & 32 & 56 & 28 \\
\hline Red meat & 198 & 99 & 2 & 1 & 0 & 0 \\
\hline White meat & 122 & 61 & 76 & 38 & 2 & 1 \\
\hline Organ meat & 198 & 99 & 2 & 1 & 0 & 0 \\
\hline Chocolate & 86 & 43 & 100 & 50 & 14 & 7 \\
\hline Herbs & 56 & 28 & 72 & 36 & 72 & 36 \\
\hline Starches & 186 & 93 & 14 & 7 & 0 & 0 \\
\hline Sugars & 186 & 93 & 4 & 2 & 10 & 5 \\
\hline Vegetables & 190 & 95 & 10 & 5 & 0 & 0 \\
\hline Iron tablets & 122 & 61 & 50 & 25 & 28 & 14 \\
\hline Fruits & 188 & 94 & 12 & 6 & 0 & 0 \\
\hline Milk & 194 & 97 & 6 & 3 & 0 & 0 \\
\hline Soft drinks & 94 & 47 & 76 & 38 & 30 & 15 \\
\hline Nuts & 54 & 27 & 58 & 29 & 90 & 45 \\
\hline Vitamins & 126 & 63 & 48 & 24 & 26 & 13 \\
\hline
\end{tabular}


Table (2): Popular health beliefs about hygiene during pregnancy

\begin{tabular}{|c|c|c|c|c|c|c|}
\hline Hygiene concepts & \multicolumn{2}{|c|}{ Agree } & \multicolumn{2}{c|}{ Equivocal } & \multicolumn{2}{c|}{ Disagree } \\
\cline { 2 - 7 } & No. & $\%$ & No. & $\%$ & No. & \% \\
\hline Bathing & 142 & 71 & 48 & 24 & 10 & 36 \\
\hline Bathing frequently & 56 & 28 & 70 & 35 & 32 & 0 \\
\hline Tooth brushing & 136 & 68 & 64 & 71 & 44 & 22 \\
\hline $\begin{array}{c}\text { Tooth brushing } \\
\text { frequently }\end{array}$ & 18 & 9 & 142 & 0 & 0 \\
\hline $\begin{array}{c}\text { Hand washing b/a } \\
\text { eating }\end{array}$ & 176 & 88 & 24 & 12 & 0 & 0 \\
\hline $\begin{array}{c}\text { Hand washing b/a } \\
\text { toilet }\end{array}$ & 192 & 96 & 8 & 4 & & 0 \\
\hline
\end{tabular}

Table (3): Popular health beliefs about physical activity during pregnancy

\begin{tabular}{|c|c|c|c|c|c|c|}
\hline \multirow{2}{*}{ Activity concepts } & \multicolumn{2}{|c|}{ Agree } & \multicolumn{2}{c|}{ Equivocal } & \multicolumn{2}{c|}{ Disagree } \\
\cline { 2 - 7 } & No. & $\%$ & No. & $\%$ & No. & $\%$ \\
\hline Walking & 72 & 36 & 92 & 46 & 36 & 18 \\
\hline Up stairs climbing & 0 & 0 & 32 & 16 & 168 & 84 \\
\hline Aerobics & 6 & 3 & 12 & 6 & 192 & 96 \\
\hline Heavy weight lifting & 0 & 0 & 0 & 0 & 200 & 100 \\
\hline Running & 6 & 3 & 2 & 1 & 192 & 96 \\
\hline
\end{tabular}

Table (4): Popular health beliefs about dressing during pregnancy.

\begin{tabular}{|c|c|c|c|c|c|c|}
\hline \multirow{2}{*}{ Type of dressing } & \multicolumn{2}{|c|}{ Agree } & \multicolumn{2}{c|}{ Equivocal } & \multicolumn{2}{c|}{ Disagree } \\
\cline { 2 - 7 } & No. & $\%$ & No. & $\%$ & No. & $\%$ \\
\hline Tight clothes & 0 & 0 & 46 & 23 & 154 & 77 \\
\hline Wide clothes & 194 & 97 & 4 & 2 & 2 & 1 \\
\hline High heel shoes & 4 & 2 & 58 & 28 & 138 & 69 \\
\hline Flat shoes & 196 & 98 & 4 & 2 & 0 & 0 \\
\hline Without shoe & 38 & 19 & 98 & 49 & 64 & 32 \\
\hline Color taboos & 4 & 2 & 192 & 96 & 4 & 2 \\
\hline Color preference & 4 & 2 & 192 & 96 & 4 & 2 \\
\hline
\end{tabular}

Table (5): Popular health beliefs about scientific medical activities during pregnancy.

\begin{tabular}{|c|c|c|c|c|c|c|}
\hline \multirow{2}{*}{ Medical service } & \multicolumn{2}{|c|}{ Agree } & \multicolumn{2}{c|}{ Equivocal } & \multicolumn{2}{c|}{ Disagree } \\
\cline { 2 - 7 } & No. & $\%$ & No. & $\%$ & No. & $\%$ \\
\hline Vaccination & 154 & 77 & 28 & 14 & 18 & 9 \\
\hline Health visits & 74 & 37 & 126 & 63 & 0 & 0 \\
\hline $\begin{array}{c}\text { Health visit } \\
\text { frequency }\end{array}$ & 24 & 12 & 84 & 42 & 92 & 46 \\
\hline X-rays & 0 & 0 & 0 & 0 & 200 & 100 \\
\hline Dentist procedures & 56 & 28 & 134 & 67 & 10 & 5 \\
\hline Medications & 4 & 2 & 16 & 16 & 180 & 90 \\
\hline $\begin{array}{c}\text { Prescribed } \\
\text { medication }\end{array}$ & 160 & 80 & 12 & 6 & 28 & 14 \\
\hline Injections & 44 & 22 & 32 & 32 & 124 & 62 \\
\hline
\end{tabular}

Table (6): Popular health beliefs about contraception.

\begin{tabular}{|c|c|c|c|c|c|c|}
\hline \multirow{2}{*}{$\begin{array}{c}\text { Types of } \\
\text { contraception used }\end{array}$} & \multicolumn{2}{|c|}{ Agree } & \multicolumn{2}{|c|}{ Equivocal } & \multicolumn{2}{|c|}{ Disagree } \\
\hline & No. & $\%$ & No. & $\%$ & No. & $\%$ \\
\hline Oral & 8 & 4 & 22 & 11 & 170 & 85 \\
\hline Injectable & 0 & 0 & 24 & 12 & 176 & 88 \\
\hline IUDs & 26 & 13 & 50 & 25 & 124 & 62 \\
\hline Segregation & 34 & 17 & 94 & 47 & 72 & 36 \\
\hline
\end{tabular}


Table (7): Popular health beliefs about delivery.

\begin{tabular}{|c|c|c|c|c|c|c|}
\hline \multirow{2}{*}{ Type } & \multicolumn{2}{|c|}{ Agree } & \multicolumn{2}{c|}{ Equivocal } & \multicolumn{2}{c|}{ Disagree } \\
\cline { 2 - 7 } & No. & $\%$ & No. & $\%$ & No. & $\%$ \\
\hline Home & 24 & 12 & 110 & 55 & 66 & 33 \\
\hline Trained dai & 48 & 24 & 118 & 59 & 34 & 17 \\
\hline Untrained dai & 10 & 5 & 40 & 20 & 150 & 75 \\
\hline Hospitals & 182 & 91 & 18 & 9 & 0 & 0 \\
\hline Colostrums & 166 & 83 & 30 & 15 & 4 & 2 \\
\hline
\end{tabular}

Table (8): Popular health beliefs about infant umbilical care.

\begin{tabular}{|c|c|c|c|c|c|c|}
\hline \multirow{2}{*}{ Types of applicants } & \multicolumn{2}{|c|}{ Agree } & \multicolumn{2}{c|}{ Equivocal } & \multicolumn{2}{c|}{ Disagree } \\
\cline { 2 - 7 } & No. & $\%$ & No. & $\%$ & No. & $\%$ \\
\hline Ash of or dung & 36 & 18 & 28 & 14 & 136 & 68 \\
\hline $\begin{array}{c}\text { Powders of medical } \\
\text { capsules }\end{array}$ & 48 & 24 & 34 & 17 & 118 & 59 \\
\hline Powders & 76 & 38 & 24 & 12 & 100 & 50 \\
\hline Ointment & 118 & 59 & 22 & 11 & 60 & 30 \\
\hline Nothing & 60 & 30 & 36 & 18 & 104 & 52 \\
\hline
\end{tabular}

Table (9): Popular health beliefs about breast feeding.

\begin{tabular}{|c|c|c|c|c|c|c|}
\hline \multirow{2}{*}{ Feeding concepts } & \multicolumn{2}{|c|}{ Agree } & \multicolumn{2}{c|}{ Equivocal } & \multicolumn{2}{c|}{ Disagree } \\
\cline { 2 - 7 } & No. & $\%$ & No. & $\%$ & No. & $\%$ \\
\hline Breast & 168 & 84 & 30 & 15 & 2 & 1 \\
\hline Bottles & 14 & 7 & 94 & 47 & 92 & 46 \\
\hline Mixed & 68 & 34 & 68 & 34 & 64 & 32 \\
\hline
\end{tabular}

Table (10): Popular health beliefs about rest after delivery.

\begin{tabular}{|l|l|l|l|l|l|l|}
\hline \multirow{2}{*}{ Rest period } & \multicolumn{2}{|c|}{ Agree } & \multicolumn{2}{c|}{ Equivocal } & \multicolumn{2}{c|}{ Disagree } \\
\cline { 2 - 7 } & No. & $\%$ & No. & $\%$ & No. & $\%$ \\
\hline $1-<2$ week & 78 & 39 & 40 & 20 & 82 & 41 \\
\hline $2-<3$ week & 128 & 64 & 38 & 19 & 34 & 17 \\
\hline $3-6$ week & 120 & 60 & 54 & 27 & 26 & 13 \\
\hline
\end{tabular}

Table (11): Popular health beliefs sexual activities during pregnancy

\begin{tabular}{|c|c|c|c|c|c|c|}
\hline \multirow{2}{*}{$\begin{array}{l}\text { Time in the } \\
\text { pregnancy }\end{array}$} & \multicolumn{2}{|c|}{ Agree } & \multicolumn{2}{c|}{ Dquivocal } & $\%$ & \multicolumn{2}{c|}{ No. } & $\%$ \\
\cline { 2 - 7 } & No. & $\%$ & No. & 45 & 96 & 48 \\
\hline $1^{\text {st }}$ trimester & 14 & 7 & 90 & 54 & 36 & 18 \\
\hline $2^{\text {nd }}$ trimester & 56 & 28 & 108 & 36 & 18 & 9 \\
\hline $3^{\text {rd }}$ trimester & 110 & 55 & 72 & 45 & 9 \\
\hline
\end{tabular}

\section{Discussion:}

In a vast, multi-ethnic, multi-religious country like Iraq, it is to be expected that there are several world-views operating at the same time in people's search for health and healing. The popular health beliefs are very crucial to the health outcome of the population and are very important for any physician to realize how their patients think, behave, and how they respond to the various therapeutic options and plans.

This research aims to gain much information regarding popular health beliefs about pregnancy in order to evaluate these beliefs according to three domain from the scientific point of view as beneficial, harmful and useless. Accordingly, special health education programs can be initiated in accordance to these results by promoting the true beliefs and managing the false beliefs by proper motivation programs.

Regarding the subject of the nutrition in the popular health beliefs, this study reveals that the majority of respondents considered that vegetables, fruits, vitamins, milk, iron tablets in addition to red, organ and white meats are beneficial to the pregnant women. These beliefs are considered good popular health beliefs that should be promoted and encouraged. Similar results were obtained by Burk et $\mathrm{al}^{(9)}$ in his study in the USA about the popular health beliefs during pregnancy. Conversely, most of the respondents in our work, regarded starches and sugars carry great benefits to the pregnant women. Moreover, considerable 
number did so regarding animal oils and soft drinks. Actually, these are harmful health beliefs carried by population and should be managed, because these beliefs are among the unhealthy western dietary behaviors that many researches proved their dangers on the health.

Most of the study participants considered bathing, tooth brushing and hand washing as good practices for the pregnant women. These beliefs, in fact, are good for health and comparable results were achieved by Guendelman ${ }^{(10)}$ according to his study in the USA on immigrants. He depicted that some pregnant women may indulge in obsessive practices about the hygiene of their bodies when they have conceived. Hygiene practices are highly related to the cultural, religious, economic and sociological backgrounds of the individual. Moreover, even the psychological entity of the individual plays an important role in his hygiene practice.

Nearly all respondents in this study, disapproved vigorous exercises (such as up stairs climbing, running, and heavy weights lifting) for the pregnant women and considered them very harmful to the health. These results go inversely with the results of Petersen ${ }^{(11)}$ who found that most of the pregnant women in the Western societies prefer many physical and sport activities during their pregnancy in order to maintain an ideal body weight and to avoid obesity. Our population beliefs seem to be very dedicated about physical exercises for the pregnant women; really, moderate physical exercises are quite helpful for the pregnant women according to Hopkins ${ }^{(12)}$ who concluded that scientifically structured minimal physical exercises are very necessary to all pregnant women, even to those with chronic diseases.

The present study shows that almost all women refused tight clothes and high heel shoes for the pregnant women. These results go in accordance with healthy dressing behavior for the pregnant women from the scientific point of view ${ }^{(13)}$. That is, in trying to avoid tear and wear effects on the weight bearing joints especially during such physically stressful periods as in pregnancy. Although, women, generally are highly attracted to follow the latest fashion models in the world, but during pregnancy, this desire is abolished for many reasons such as the stress of pregnancy, and the relatively short period of pregnancy. In addition to that, the purdah system in most of Islamic countries, makes the models not so important.

Significant number of participants in this effort, have good popular health beliefs by their agreement with vaccination for the pregnant women and the taking of prescribed medication in addition to their refusal to exposure of the pregnant women to any form of X-rays or non-prescribed medications. In fact these are good filtered information from the professional health sectors and media that should be promoted and reinforced. But, their beliefs about regular health visit to the available health facilities and to the dentists were quite unreliable and unhelpful. This requires proper interventional educational programs, because many health problems during pregnancy can be simply managed by regular health visits to the available health facilities. Moreover, many other health complications can be prevented. Burk et $\mathrm{al}^{5}$, in his study about Mexican- American women, found that nearly half of the participants (49\%)in that study were unaware about the health facilities that were available for them. Moreover, about three quarters of them $(71 \%)$ regarded dental problems are among the subjects that should be postponed beyond delivery. Such popular beliefs should be managed not only by proper health education programs, but by proper health advertising strategy through media.

The majority in this study disagreed about any form of contraception and this relied heavily on the religious and other socio-cultural variables of our population and explaining partly the elevated growth rates in our locality ${ }^{(14)}$. In most western societies, according to many factors related to the cultural and religious backgrounds, different modalities of contraceptives are available and being in use by large proportion of women, and this has led to many health problems. Unger and Monila ${ }^{(14)}$ found that the rate of use of contraceptives increased many folds among immigrants to the USA in relation to their background population. They attributed this increase to the process of acculturation and major changes in the beliefs especially of the new generation that are born in different societies to their original families. Nevertheless, this dissimilarity between western and eastern societies is among many other differences 
that are related to religious, cultural and sociological backgrounds.

The present study demonstrated that a large number $(91 \%)$ approved deliveries at hospitals and confirmed the use of colostrum to the newly born babies. Parallel results were found by Mendelson ${ }^{(15)}$ in his study in the USA where the majority $(89 \%)$ of the participants agreed and promoted the use of colostrum as a food to the newly born babies soon after deliveries. In addition, more than half $(52 \%)$ preferred breast feeding. Similar results were found by Al- Naemi ${ }^{(22)}$ in her study in Mosul city, where $(97.8 \%)$ of the participants in that study regarded breast feeding is the ideal feeding practice to the infants.

Greater part among participants (84\%) were in agreement with breast feeding alone for the newly born babies, but about one third of them expressed also inconsistency with mixed type of feeding. Parallel results were found among many studies such as that of Mendoza and Peterson ${ }^{16}$; and Kramer et $\mathrm{al}^{(17)}$, where participants in their studies agreed about breast feeding by $(79 \%)$ and $(76 \%)$ respectively. This popular health belief is excellent and beneficial to the health of the new born babies. By the effect of rapid modernization and globalization, the increasing attitude towards bottle feeding of new born is found to be very harmful to the health of both the mother and her baby ${ }^{(19)}$.

About one fifth of participants in this work agreed about the risky procedure of applying dung or ash of dung to the umbilical stump. In fact, this result can explain the still occurring very dangerous tetanus neonatorum. This critical popular belief must be taken into account urgently. This tragedian fact, had been reported in different societies all over the world and it is among the most dangerous bad health beliefs that is carried out, till now, by lay people ${ }^{(18)}$.

After birth, women in most cultures observe a special postpartum rest period, during which they have to follow certain dietary and other taboos and are cared for mainly by other women. Sometime there are special rituals which purify the house, as well as a ritual bath for the mother and the shaving of the baby's head. A further important aspect of the postpartum period is that many cultures prohibit sexual relations between husband and wife for a period of time after birth. In some cases this may last for several months as in China ${ }^{(20)}$. In this study, about two thirds of the respondents $(64 \%)$ preferred more than two weeks rest after delivery for the pregnant women and $(60 \%)$ of them wished to get up to 40 days as a rest period after delivery.

The childbirth for a woman is more than just a medical condition, it emphasizes the acquiring (with the first birth) a new social role of the mother, as well as profound changes in her financial situation, marital status, housing situation and personal relationships. Therefore, in all human societies pregnancy and childbirth are more than just biological events. They are also part of an important transition of the woman from the social state of "woman" to that of "mother". As with all social transitions, during the dangerous journey from one status to another, the individual must be "protected" from harm by the observance of certain ritual beliefs and behavior. In many of these transitions the person concerned goes through a temporary period of "withdrawal" from ordinary life, before being "re-born" into their new social status ${ }^{(21)}$. Approximately have of interviewees in this work favored sexual activity for the pregnant women in their third trimester, but disagreed about that in the first trimester, and this partly could be explained as a sign of withdrawal that any woman may pass through it during her pregnancy.

A lot of important informations were achieved from this research about popular health beliefs about pregnancy, many of them, were found to be true and should be reinforced, however, few others were found to be harmful to the health of the pregnant women (such as the preference of sugars and starches) and to their babies (such as the application of dung to the umbilical stump) which required an urgent intervention health educational programs.

\section{References:}

1. Lipson J G, Dibble S L, \& Minarik P A (Eds.). Culture \& Nursing Care: A Pocket Guide. San Francisco: UCSF Nursing Press. 1996;p.234-237.

2. Paulanka B J, \& Purnell, L D (Eds.). Trans-cultural Healthcare: A Culturally Competent Approach. (CD-ROM). F. A. Davis Company. 1998; p.82-83.

3. Al-Shahri M Z. Culturally Sensitive Caring for Saudi Patients. Journal of 
Transcultural Nursing 2002; 13 (2): 133-138.

4. Lawrence $P$ and Rozmus C. Culturally Sensitive Care of the Muslim Patient. Journal of Transcultural Nursing 2001; 12(3): 228-233.

5. Burk M, Wieser P, Keegan L. Cultural beliefs and health behaviors of pregnancy Mexican-American women: implications for primary care. Advances in Nursing Science 1995;17(4):37-52.

6. Your Pregnancy \& Birth, Fourth Edition. The American College of Obstetricians and Gynecologists (ACOG) and Meredith Books, 2005;p24-35.

7. Andrews, J.D. "Native Americans." Cultural, Ethnic, and Religious Reference Manual for Health Care Providers. (3rd edition). WinstonSalem, N.C.: JAMARDA Resources, Inc. 2005; 111-120.

8. Amaro $\mathrm{H}$. Women in the MexicanAmerican community: religion, culture, and reproductive attitudes and experiences. Journal of Community Psychology 1988; 16: 6-20.

9. Burk $M$, Wieser $P$ and Keegan $L$. Cultural beliefs and health behaviors of pregnancy Mexican-American women: implications for primary care. Advances in Nursing Science 1995; 17(4):37-52.

10. Guendelman S. Immigrants may hold the clues to protection of health during pregnancy: Exploring a paradox. Wellness Series: 47-75, 1995.

11. Petersen A. E. Correlate of Physical Activity among Pregnant Women in the United States. Medicine \& Science in Sports \& Exercise; 2005; p.1748-1753.

12. Hopkins FW. Pregnancy-Related Mortality in Hispanic Women in the United States. Obstetrics \& Gynecology 1999; 94(5):745-752.

13. Borrayo EA and Jenkins SR. Feeling frugal: socioeconomic status, acculturation, and cultural health beliefs among women of Mexican descent. Cultur Divers Ethnic Minor Psychol 2003; 9: 197-206.

14. Unger JB and Molina GB. Acculturation and attitudes about contraceptive use among Latina women. Health Care for Women International 2000; 21:235-249.

15. Mendelson C. Health perceptions of Mexican American women. J Transcultur Nurs 2002; 13: 210-17.

16. Mendoza $M$ and Peterson MC. New Latino immigration to Tennessee: practicing culturally sensitive health care. Tenn Med 2000; 10: 371-6.

17. Kramer EJ, Tracy LC and Ivey SL. Demographics, Definitions, and Data Limitations. In EJ Kramer, SL Ivey, \& Y$W$ Ying (Eds.), Immigrant Women's Health: Problems and Solutions. San Francisco, CA: Jossey-Bass, Inc. 1995;p.85-92.

18. Mac Cormack C P. Biological, cultural and social adaptation in human fertility and birth: a synthesis. In: Ethnography of Fertility and Birth ( C P McCormack, ed.), pp. 1-23. 1982. Academic Press.

19. World Health Organization. The health of the family: some key issues. World Health Statistics Quarterly 1985; 38: 254-5. WHO.

20. Hahn R A and Muecke M A. The anthropology of birth in five U.S. ethnic population: implications for obstetrical practice. Curr. Probl. Obstet. Gynecal. Fertil.1987;10: 133-71.

21. Kitzinger $S$. The social context of birth: some comparisons between childbirth in Jamaica and Britain. In: Ethnography of Fertility and Birth (C.P. McCormack, ed.), Academic Press 1982 pp. 181203.

22. AL-Naemi M A.Evaluation of breast feeding practice in Mosul city. M.Sc. Thesis, Dep. Comm. Med., coll. Med. Univ. Mosul. P 55-58. 